\title{
Regression of Papillary Thyroid Cancer during Nivolumab for Renal Cell Cancer
}

\author{
Andrea Palermo ${ }^{a}$ Andrea Napolitano ${ }^{b}$ Daria Maggi ${ }^{a} \quad$ Anda Mihaela Naciu $^{a}$ \\ Gaia Tabacco $^{a}$ Silvia Manfrini $^{a} \quad$ Anna Crescenzi ${ }^{c}$ Chiara Taffon ${ }^{c}$ \\ Francesco Pantano $^{b}$ Bruno Vincenzi ${ }^{b}$ Guiseppe Tonini ${ }^{b}$ Daniele Santinib \\ a Unit of Endocrinology and Diabetes, Campus Bio-Medico University, Rome, Italy; ${ }^{b}$ Department of Medical \\ Oncology, Campus Bio-Medico University, Rome, Italy; ' ${ }^{\complement}$ Department of Anatomic Pathology, Campus Bio-Medico \\ University, Rome, Italy
}

\section{Established Facts}

- It is already known that papillary thyroid cancer arising in a background of Hashimoto thyroiditis shows increased programmed cell death-1 (PD-L1).

- It is already known that metastases to the thyroid gland are uncommon, but frequently associated with renal cancer, especially when presenting as a fast growing nodule.

\section{Novel Insights}

- Our case strongly supports the evaluation of nivolumab and possibly other forms of immune therapy in the treatment of papillary thyroid carcinoma.

- The presence of histiocyte aggregates on the site where the prior nodule had been observed supports the hypothesis of an immune-mediated tumor regression.

\section{Keywords}

Cancer · Thyroid cancer · Nivolumab · Immune checkpoint inhibitors

\section{Abstract}

Immune checkpoint inhibitors have been recently approved for cancer treatment. Nivolumab is a monoclonal antibody specific for programmed cell death-1 (PD-1) that modulates T-cell response. It was initially used for the treatment of ma- lignant melanoma and then approved in other cancers, such as non-small cell lung cancer and clear cell renal cell carcinoma (ccRCC). So far, the activity of nivolumab in patients with thyroid malignancies has been reported in a single case of anaplastic thyroid cancer. Here, we report the case of a patient with ccRCC who developed a papillary thyroid carci-

Andrea Palermo and Andrea Napolitano contributed equally to this work. 
noma (PTC) under first-line sunitinib treatment. During nivolumab, the second-line treatment for $\mathrm{cCRCC}$, we unexpectedly observed a complete regression of PTC.

(C) 2020 European Thyroid Association Published by S. Karger AG, Basel

\section{Introduction}

Immune checkpoint inhibitors have been recently approved for cancer treatment. Programmed cell death-1 (PD-1) and cytotoxic T-lymphocyte antigen 4 (CTLA-4) are checkpoint proteins expressed on the surface of cytotoxic T-cells which interact with their ligands to regulate immune response in physiologic conditions. Monoclonal antibodies that target either PD-1 or its ligands (e.g., PDL1) can restore the host immune reaction against cancer cells [1]. Nivolumab is a monoclonal antibody specific for PD-1 that modulates T-cell response. It was initially used for the treatment of malignant melanoma and then approved in other cancers, such as non-small cell lung cancer and clear cell renal cell carcinoma (ccRCC) [2]. More recently, a case of anaplastic thyroid cancer treated with vemurafenib and nivolumab has been described, achieving substantial regression and complete radiographic and clinical remission [3].

\section{Case Report}

In February 2014, a 54-year-old male was referred to our center, 1 month after resection of a right ccRCC pT1a, with evidence of two suspect osteolytic lesions localized in the L1 and L2 vertebrae. A bone biopsy was performed, confirming the presence of carcinoma cells compatible with ccRCC. The prognostic Motzer risk score, which is used to predict patient prognosis based on clinical and hematological parameters [4], was intermediate (i.e., around 10-12 months). In March, the patient received antalgic radiation therapy to L1 and L2 for a total dose of $36 \mathrm{~Gy}$ and was then started on first-line sunitinib $50 \mathrm{mg}$ per day (4-weeks-on/2weeks-off schedule) and zoledronic acid. In April 2014, due to G2 asthenia and G2 hand-foot syndrome, the sunitinib schedule was switched to 2-weeks-on/1-week-off, with better tolerability. In February 2015, due to worsening of hand-foot syndrome, the dose of sunitinib was reduced to $37.5 \mathrm{mg}$ per day (Fig. 1), which the patient continued until November 2017 due to stability of the bone lesions at periodic CT and MRI scans.

As often observed during treatment with sunitinib [5], in November 2014 the patient presented subclinical hypothyroidism (Fig. 1), with negative antibodies against thyroglobulin and thyroperoxidase. No L-thyroxine replacement therapy was initiated. The neck ultrasound (US) showed a thyroid gland of normal volume, without nodules or parenchymal abnormalities. Serological values of TSH normalized without therapy in March 2015, after reduction of the sunitinib dose. In July 2016, the follow-up neck US showed a thyroid micronodule $(4 \times 4 \times 5 \mathrm{~mm}$, vol $0.042 \mathrm{~mL})$ solid in nature, strongly

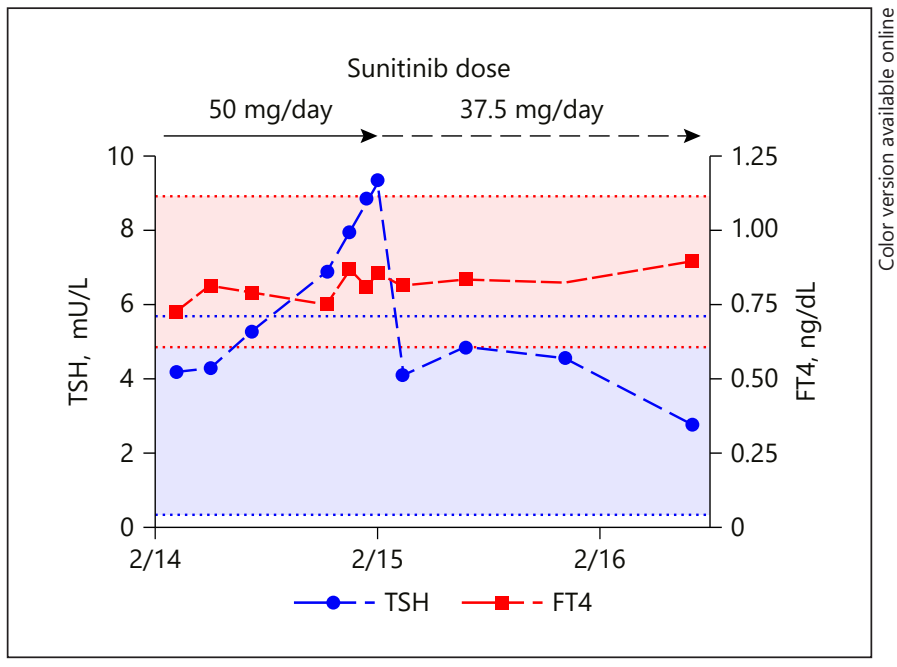

Fig. 1. Timeline of sunitinib treatment and subclinical hypothyroidism. Blue and red horizontal lines and areas delimitate normal values for TSH and FT4, respectively.

hypoechoic, with taller-than-wide shape, ill-defined margins, and little peripheral vascularity on Doppler US. Based on the small dimensions of the nodule, periodic US follow-up was scheduled.

In October 2017, spine MRI scans showed appearance of a suspect metastatic bone lesion in $\mathrm{C} 4$ vertebra. Bone scintigraphy, performed in November 2017, confirmed tracer hyper-fixation in the cervical spine. The patient refused radiation therapy, and secondline therapy with nivolumab was planned for December 2017.

Before starting nivolumab, the patient underwent follow-up neck US, in which a significant volumetric increase in the nodule was detected (dimensions of $14 \times 10 \times 18 \mathrm{~mm}$, vol $1.3 \mathrm{~mL}$ ) (Fig. 2a), and fine needle aspiration was performed. Cytological smears showed scanty atypical thyrocytes in a bloody background. These cells had overlapping oval nuclei with grooves (Fig. 2b) and were positive for thyroid transcription factor 1 (TTF1) immunostaining (Fig. 2c). The diagnosis was of cytology suspicious for papillary thyroid carcinoma (PTC), category TIR4 according to the Italian consensus for the classification and reporting of thyroid cytology [6]. Based on the cytology and the radiological evolution of the lesion, a diagnosis of PTC was made. After a multidisciplinary meeting, in consideration of the progressive bone disease secondary to ccRCC, it was decided to postpone thyroidectomy and to start treatment with nivolumab as planned.

Nivolumab was started in December 2017, with good tolerability. In March 2017, a minimally progressive disease was recorded (appearance of small non-painful bone lesions in D9, D11, and D12 vertebrae), but treatment was continued beyond progression in absence of cancer-related symptoms. At the last available scans (November 2018), the bone lesions were stable in dimension. Treatment with nivolumab is still ongoing.

At the 6-month follow-up visit after detection of PTC (i.e., in July 2017 after 6 months of nivolumab therapy), the neck US showed a thyroid gland with slightly irregular texture, without clear evidence of the previously diagnosed nodule. In order to confirm the regression of the lesion, a thyroid core biopsy was performed on the site where the prior nodule had been observed. Mi- 

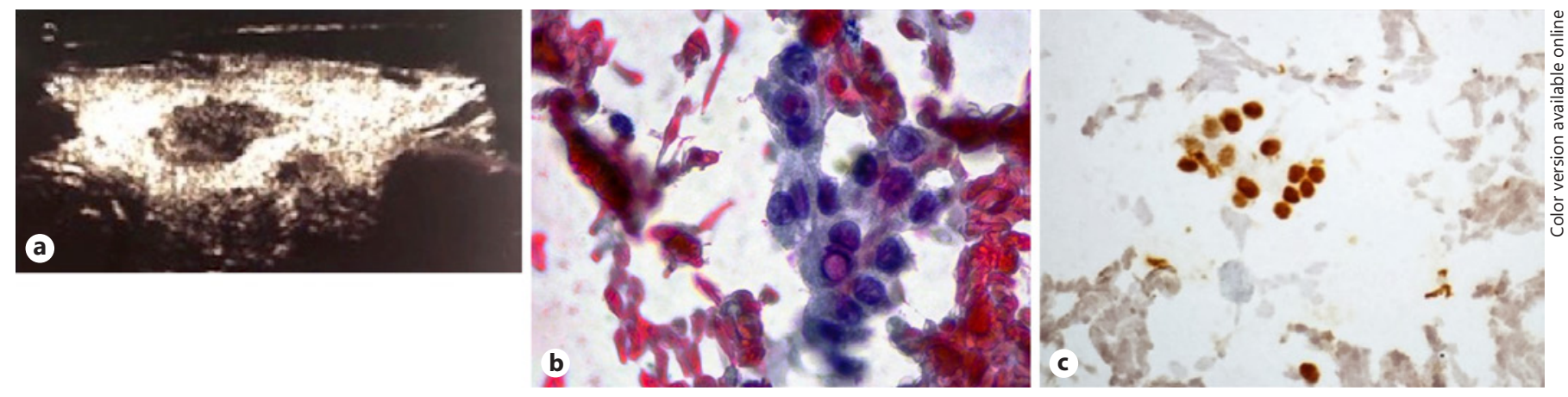

Fig. 2. Characteristics of the nodule; pre-nivolumab treatment. a Neck US findings. Solid, strong hypoechoic thyroid nodule with ill-defined margin. b Nodule FNA cytology. Sparse groups of atypical thyrocytes with nuclear grooves and pseudoinclusion. High-power field. Papanicolaou stain. c Cytological smears stained with immunohistochemistry for TTF1. Positive reaction in brown in the irregular nuclei supports the thyroid origin of the lesion. High-power field.
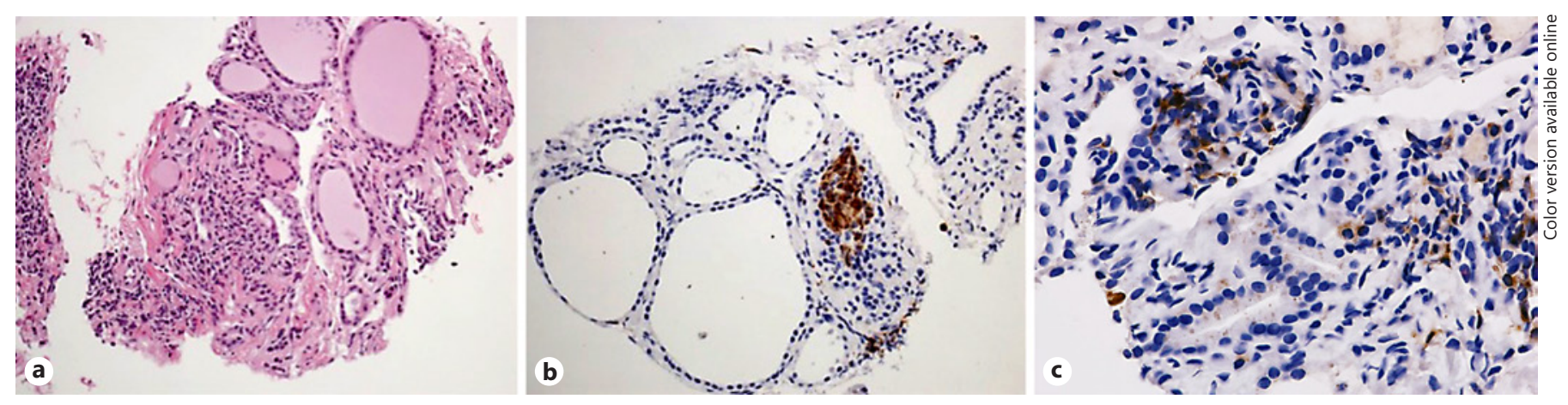

Fig. 3. Characteristics of nodule; post-nivolumab treatment. a Thyroid core biopsy: hematoxylin/eosin. Normal follicular architecture with lymphocytic infiltrate and thin collagen bundles and initial fibrosis. Medium-power field. b Thyroid core biopsy: CD68 (histiocyte marker) immunohistochemistry. Aggregate of brown positive cells within the thyroid parenchyma. Medium-power field. c CD4 immunohistochemistry. Lymphocytes, subtype of T-cells, and histiocytes show positive stain. Medium-power field.

croscopic evaluation of the core showed a thyroid parenchyma with normal follicles and lymphomonocytic infiltrate, as observed in thyroiditis (Fig. 3a). An area of focal cellular reaction with histiocyte aggregates was observed and confirmed by immunohistochemistry for CD68 (Fig. 3b). Immunostaining for CD4 and CD8 showed CD4-positive lymphocytes and histiocytes (Fig. 3c), without relevant presence of CD8-positive lymphocytes. The final diagnosis was chronic thyroiditis with focal granulomatous features and no residual cancer. Follow-up neck US, as well as TSH and FT4 values have been stable within normal limits so far.

\section{Discussion}

The association between a first diagnosis of ccRCC and a subsequent thyroid cancer is strong and often underrecognized by clinicians. Although, as in our case, it might be partially explained as a result of continued surveillance bias [7]. Metastases to the thyroid gland are uncommon, but frequently associated with renal cancer, especially when presenting as a fast growing nodule as in our case [8]. For this reason, we performed TTF1 immunostaining on the cytological sample, which confirmed the thyroidal origin.

PTC accounts for approximately $85 \%$ of thyroid cancers and is generally associated with a good prognosis. Treatment modalities most often include surgery and radioiodine therapy. Systemic therapies with tyrosine kinase inhibitors are reserved for metastatic radioiodineresistant cases [9]. Interestingly, in our patient, PTC was originally diagnosed when the patient was under treatment with the tyrosine kinase inhibitor sunitinib, possibly an even rarer occurrence. In animal models, TSH 
stimulation contributes to the development of thyroid cancer, and high serum TSH has been associated with higher risk of thyroid cancer in patients with thyroid nodules [10]. It is difficult to establish whether fluctuating levels of TSH over time might have influenced the development and growth of PTC in our case.

Since tyrosine kinase inhibitors as well as immune checkpoints inhibitors may induce thyroid dysfunction and thyroiditis $[11,12]$, cytopathologists should carefully consider the interpretation of nuclear changes, such as clearing or membrane irregularities, mimicking papillary carcinoma. In thyroiditis, however, nuclear alterations are usually mild and focal. In our case, well-developed papillary nuclear features were observed in bloody, paucicellular smears. Due to this limiting factor, the pathological report was classified as suspicious for papillary carcinoma.

The therapeutic effect of nivolumab monotherapy in ccRCC has been reported in phase III clinical trials. In this study, nivolumab showed a significantly longer overall survival compared to the mTOR inhibitor everolimus [13]. On the contrary, the use of checkpoint inhibitors is not part of the standard therapeutic algorithm for PTC [14]. Positivity of PD-L1 has been in fact consistently reported in PTC, especially in aggressive cases [15-20], as well as in a subset of patients with advanced follicular and anaplastic thyroid cancers [21]. In particular, PTC arising in a background of Hashimoto thyroiditis shows increased PD-L1 expression [22]. Whether the sunitinibinduced thyroid dysfunction might be associated with increased PD-L1 expression is only speculative. Although our patient was eventually diagnosed with a chronic thyroiditis, autoantibodies were negative during the diagnostic workup. The presence of histiocyte aggregates supports the hypothesis of an immune-mediated tumor regression. Other potential biomarkers predictive of response to checkpoint inhibitors have also been assessed in PTC: the tumor mutation burden is generally low consistent with what is observed with other endocrine tumors - although immunogenic mutation might still be present [23]. Finally, the presence of a lymphocytic infiltrate in PTC has shown controversial prognostic associations, with most reports, however, suggesting an anti-tumoral role of the inflammatory milieu $[24,25]$.

From a clinical perspective, the case of a patient with anaplastic thyroid carcinoma responding to nivolumab treatment was recently reported [3]. Ongoing trials are still formally evaluating the potential role of immunotherapy in advanced thyroid cancers; specifically, nivolumab is being tested in a phase II trial alone or in combination with ipilimumab (a CTLA-4 inhibitor) in metastatic, radioiodine-refractory, differentiated thyroid cancers (NCT03246958). Our case strongly supports the evaluation of nivolumab and possibly other forms of immune therapy in the treatment of PTC.

\section{Statement of Ethics}

Ethics approval and consent to participate: not applicable. Consent for publication: an institutional consent form for data publication was obtained from the patient.

\section{Disclosure Statement}

The authors have no conflicts of interest to declare.

\section{Funding Sources}

No funding was received for the writing of this paper.

\section{Author Contributions}

A.P. and A.N.: patient management, figure preparation, manuscript writing. D.M. and S.M.: patient management. A.M.N. and G.T.: patient management, manuscript writing. A.C. and C.T.: pathological analysis. F.P.: patient management, manuscript writing. G.T.: patient management, manuscript editing. D.S.: patient management, manuscript writing, manuscript editing.

\section{References}

1 Postow MA, Callahan MK, Wolchok JD. Immune Checkpoint Blockade in Cancer Therapy. J Clin Oncol. 2015 Jun;33(17):1974-82.

2 BrahmerJR, Hammers H, Lipson EJ. Nivolumab: targeting PD-1 to bolster antitumor immunity. Future Oncol. 2015;11(9):1307-26.

3 Kollipara R, Schneider B, Radovich M, Babu S, Kiel PJ. Exceptional Response with Immu- notherapy in a Patient with Anaplastic Thyroid Cancer. Oncologist. 2017 Oct;22(10): 1149-51.

4 Motzer RJ, Mazumdar M, Bacik J, Berg W, Amsterdam A, Ferrara J. Survival and prognostic stratification of 670 patients with advanced renal cell carcinoma. J Clin Oncol. 1999 Aug;17(8):2530-40.
5 Rini BI, Tamaskar I, Shaheen P, Salas R, Garcia J, Wood L, et al. Hypothyroidism in patients with metastatic renal cell carcinoma treated with sunitinib. J Natl Cancer Inst. 2007 Jan;99(1):81-3.

6 Nardi F, Basolo F, Crescenzi A, Fadda G, Frasoldati A, Orlandi F, et al. Italian consensus for the classification and reporting of thyroid cytology. J Endocrinol Invest. 2014 Jun;37(6):593-9. 
7 Lal G, Groff M, Howe JR, Weigel RJ, Sugg SL, Lynch CF. Risk of subsequent primary thyroid cancer after another malignancy: latency trends in a population-based study. Ann Surg Oncol. 2012 Jun;19(6):1887-96.

8 Nixon IJ, Coca-Pelaz A, Kaleva AI, Triantafyllou A, Angelos P, Owen RP, et al. Metastasis to the Thyroid Gland: A Critical Review. Ann Surg Oncol. 2017 Jun;24(6):1533-9.

9 Fagin JA, Wells SA Jr. Biologic and Clinical Perspectives on Thyroid Cancer. N Engl J Med. 2016 Sep;375(11):1054-67.

10 Fiore E, Vitti P. Serum TSH and risk of papillary thyroid cancer in nodular thyroid disease. J Clin Endocrinol Metab. 2012 Apr;97(4): 1134-45.

11 Illouz F, Braun D, Briet C, Schweizer U, Rodien P. Endocrine side-effects of anti-cancer drugs: thyroid effects of tyrosine kinase inhibitors. Eur J Endocrinol. 2014 Sep;171(3):R919.

12 Iyer PC, Cabanillas ME, Waguespack SG, Hu MI, Thosani S, Lavis VR, et al. Immune-Related Thyroiditis with Immune Checkpoint Inhibitors. Thyroid. 2018 Oct;28(10):124351.

13 Motzer RJ, Escudier B, McDermott DF, George S, Hammers HJ, Srinivas S, et al.; CheckMate 025 Investigators. Nivolumab versus Everolimus in Advanced Renal-Cell Carcinoma. N Engl J Med. 2015 Nov;373(19): 1803-13.
14 Antonelli A, Ferrari SM, Fallahi P. Current and future immunotherapies for thyroid cancer. Expert Rev Anticancer Ther. 2018 Feb; 18(2):149-59.

15 Chowdhury S, Veyhl J, Jessa F, Polyakova O, Alenzi A, MacMillan C, et al. Programmed death-ligand 1 overexpression is a prognostic marker for aggressive papillary thyroid cancer and its variants. Oncotarget. $2016 \mathrm{May} ; 7(22)$ : 32318-28.

16 Kim K, Jeon S, Kim TM, Jung CK. Immune Gene Signature Delineates a Subclass of Papillary Thyroid Cancer with Unfavorable Clinical Outcomes. Cancers (Basel). 2018 Dec; 10(12):E494.

17 Tuccilli C, Baldini E, Sorrenti S, Catania A, Antonelli A, Fallahi P, et al. CTLA-4 and PD-1 Ligand Gene Expression in Epithelial Thyroid Cancers. Int J Endocrinol. 2018 Jul; 2018:1742951.

18 An HJ, Ko GH, Lee JH, Lee JS, Kim DC, Yang JW, et al. Programmed Death-Ligand 1 Expression and Its Correlation with Lymph Node Metastasis in Papillary Thyroid Carcinoma. J Pathol Transl Med. 2018 Jan;52(1): 9-13.

19 Fu G, Polyakova O, MacMillan C, Ralhan R, Walfish PG. Programmed Death - Ligand 1 Expression Distinguishes Invasive Encapsulated Follicular Variant of Papillary Thyroid Carcinoma from Noninvasive Follicular Thyroid Neoplasm with Papillary-like Nuclear Features. EBioMedicine. 2017 Apr;18:50-5.
20 Shi RL, Qu N, Luo TX, Xiang J, Liao T, Sun $\mathrm{GH}$, et al. Programmed Death-Ligand 1 Expression in Papillary Thyroid Cancer and Its Correlation with Clinicopathologic Factors and Recurrence. Thyroid. 2017 Apr;27(4): 537-45.

21 Ahn S, Kim TH, Kim SW, Ki CS, Jang HW, Kim JS, et al. Comprehensive screening for PD-L1 expression in thyroid cancer. Endocr Relat Cancer. 2017 Feb;24(2):97-106.

22 Lubin D, Baraban E, Lisby A, Jalali-Farahani S, Zhang P, Livolsi V. Papillary Thyroid Carcinoma Emerging from Hashimoto Thyroiditis Demonstrates Increased PD-L1 Expression, Which Persists with Metastasis. Endocr Pathol. 2018 Dec;29(4):317-23.

23 Pozdeyev N, Gay LM, Sokol ES, Hartmaier R, Deaver KE, Davis S, et al. Genetic Analysis of 779 Advanced Differentiated and Anaplastic Thyroid Cancers. Clin Cancer Res. 2018 Jul; 24(13):3059-68.

24 Aghajani MJ, Yang T, McCafferty CE, Graham S, Wu X, Niles N. Predictive relevance of programmed cell death protein 1 and tumorinfiltrating lymphocyte expression in papillary thyroid cancer. Surgery. 2018 Jan;163(1): 130-6.

25 Pilli T, Toti P, Occhini R, Castagna MG, Cantara S, Caselli M, et al. Chronic lymphocytic thyroiditis (CLT) has a positive prognostic value in papillary thyroid cancer (PTC) patients: the potential key role of Foxp $3+$ T lymphocytes. J Endocrinol Invest. 2018 Jun;41(6):703-9. 